\title{
Altered miRNA expression profiles are involved in the protective effects of troxerutin against ultraviolet $B$ radiation in normal human dermal fibroblasts
}

\author{
HWA JUN CHA ${ }^{1}$, KWANG SIK LEE ${ }^{2,3}$, GHANG TAI LEE ${ }^{2,3}$, KUN KOOK LEE $^{2}$, JIN TAE HONG ${ }^{3}$, \\ SUNG NAE LEE ${ }^{4}$, HYUN HEE JANG ${ }^{5}$, JAE HO LEE ${ }^{6}$, IN-CHUL PARK ${ }^{7}$, YU RI KIM ${ }^{8}$, KYU JOONG AHN ${ }^{8}$, \\ SEUNG BIN KWON ${ }^{1}$, IN-SOOK AN ${ }^{1}$, SUNGKWAN AN ${ }^{1}$ and SEUNGHEE BAE ${ }^{1}$ \\ ${ }^{1}$ Korea Institute for Skin and Clinical Sciences and Molecular-Targeted Drug Research Center, Konkuk University, \\ Seoul 143-701; ${ }^{2}$ Songpa R\&D Center, Coreana Cosmetics Co., Ltd., Cheonan, Chungcheongnam-do 330-833; \\ ${ }^{3}$ College of Pharmacy and Medical Research Center, Chungbuk National University, Cheongju, \\ Chungcheongbuk-do 361-763; ${ }^{4}$ Department of Cosmetology, Kyung-In Women's College, Incheon 407-740; \\ ${ }^{5}$ School of Art, Kyungbok University, Namyangju, Gyeonggi-do 472-948, ${ }^{6}$ Laboratory of Molecular Oncology, \\ Cheil General Hospital and Women's Healthcare Center, Kwandong University College of Medicine, Seoul 100-380; \\ ${ }^{7}$ Laboratory of Functional Genomics, Korea Institute of Radiological and Medical Sciences, Seoul 139-706; \\ ${ }^{8}$ Department of Dermatology, Konkuk University School of Medicine, Seoul 143-701, Republic of Korea
}

Received September 23, 2013; Accepted January 28, 2014

DOI: $10.3892 / \mathrm{ijmm} .2014 .1647$

\begin{abstract}
The aim of this study was to investigate the mechanisms by which troxerutin protects cells against ultraviolet $\mathrm{B}$ (UVB) radiation. First, we demonstrate that pre-treatment with troxerutin protects normal human dermal fibroblasts (nHDFs) against UVB-induced cytotoxicity. As shown by migration assay and DNA repair analysis, troxerutin increased cell migration and DNA repair activity in the nHDFs. Subsequently, we analyzed microRNA (miRNA) expression profiles in the nHDFs. miRNAs are 19- to 24-nucleotide (nt) non-coding RNA molecules that regulate the translation of target genes through RNA interference. In UVB-exposed cells, miRNAs act on crucial functions, such as apoptosis and cellular senescence. miRNA expression is significantly altered during the protective process induced by phytochemicals. Therefore, understanding changes that occur in miRNA expression profiles may help to elucidate the protective mechanisms of troxerutin. We identified 11 miRNAs that were significantly ( $>2$-fold) upregulated and 12 that were significantly downregulated ( $>2$-fold) following treatment of the nHDFs with troxerutin. In addition, we investigated the biological functions of these miRNAs through the prediction of miRNA targets and Gene Ontology analysis of the
\end{abstract}

Correspondence to: Professor Seunghee Bae, Korea Institute for Skin and Clinical Sciences and Molecular-Targeted Drug Research Center, Konkuk University, 120 Neungdong-ro, Gwangjin-gu, Seoul 143-701, Republic of Korea

E-mail: sbae@konkuk.ac.kr

Key words: troxerutin, normal human dermal fibroblasts, ultraviolet B, microRNA, cell death putative targets. Overall, our findings indicate that pre-treatment with troxerutin increases the viability of UVB-exposed nHDFs through the alteration of the miRNA expression profiles.

\section{Introduction}

In the dermis, fibroblasts that are derived from mesenchymal cells play a critical role in maintaining homeostasis $(1,2)$. Dermal fibroblasts synthesize and secrete extracellular matrix (ECM) components, such as collagen and elastin (3). The ability to synthesize ECM components is repressed by chronic and acute solar ultraviolet (UV) exposure $(4,5)$, and UV radiation also indirectly causes the destruction of the ECM through the induction of matrix metalloproteinase (MMP) secretion by dermal fibroblasts. The impaired ECM forms a non-elastic matrix in the dermis, which leads to aging (6). Furthermore, UV triggers apoptosis and growth arrest in fibroblasts $(7,8)$. Therefore, as dermal fibroblasts play an important role in the construction and homeostasis of the dermis and UV induces a decrease in the number of dermal fibroblasts and the destruction of the ECM, UV radiation is one of the major inducers of disorders of the dermis.

microRNAs (miRNAs) are 19- to 24-nucleotide (nt) noncoding RNA sequences that regulate target gene expression through RNA interference. miRNAs have been shown to modulate various cellular processes, such as apoptosis, proliferation, cell cycle progression and differentiation (9). Several lines of evidence suggest that miRNAs play important roles in the dermis. miRNA-152 and miRNA-181 act on adhesion proteins and ECM proteins in senescent dermal fibroblasts (13). The expression of miRNA-92a leads to a decreased expression of MMP1 and the accumulation of collagen (14), and miRNA-196a and miRNA-150 directly target collagen type I in scleroderma 
dermal fibroblasts (15). Moreover, the expression of miRNAs is sensitively altered in response to UV radiation (10-12). In a recent study, we demonstrated that phytochemicals protect various cell types against UV radiation, suggesting a role for miRNAs in this protective mechanism in dermal fibroblasts (16). However, little is known about the underlying mechanisms.

Troxerutin is a trihydroxyethylated derivative of the natural bioflavonoid, rutin, which is one of the phytochemical components extracted from Sophora japonica (17). In addition, as troxerutin has been shown to have radioprotective, anti-inflammatory and antioxidant effects, it seems likely that troxerutin may protect cells against UV-induced oxidative stress and DNA damage $(18,19)$.

In this study, we aimed to determine whether troxerutin induces a resistance response in dermal fibroblasts exposed to UVB radiation and, if so, to determine whether miRNAs are involved in the protective effects of troxerutin against UVB radiation. We confirm that the treatment of human dermal fibroblasts (HDFs) with troxerutin induces resistance to UVB-induced cell damage. In addition, we characterize the miRNA expression profiles associated with the protective effects of troxerutin against UVB radiation.

\section{Materials and methods}

Cell lines and cell culture. Normal HDFs (nHDFs) were purchased from Lonza (Basel, Switzerland) and cultured in Dulbecco's modified Eagle's medium (DMEM; GibcoInvitrogen, Carlsbad, CA, USA) supplemented with $10 \%$ fetal bovine serum (FBS; Sigma-Aldrich, St. Louis, MO, USA) and $1 \%$ penicillin/streptomycin (Gibco Invitrogen) in a humidified atmosphere of $95 \%$ air $/ 5 \% \mathrm{CO}_{2}$ at $37^{\circ} \mathrm{C}$.

WST-1 assay. Cell viability was determined using a watersoluble tetrazolium salt-1 (WST-1) assay. nHDFs were seeded in a 96-well plate (SPL Life Sciences, Pocheon, Korea) at a density of $4 \times 10^{3}$ cells/well. The nHDFs were incubated for $24 \mathrm{~h}$ under growth conditions and then pre-treated with the indicated concentrations of troxerutin for 4,8 and $12 \mathrm{~h}$. At each time point, the cells were exposed to $100 \mathrm{~mJ} / \mathrm{cm}^{2} \mathrm{UVB}$ radiation using a G8T5E lamp (Sankyo Denki, Toshima-ku, Japan) and a UV light meter (Lutron UV-340; Lutron Electronic Enterprise Co., Taipei, Taiwan). Following exposure to UVB radiation, the $\mathrm{nHDF}$ were incubated for $24 \mathrm{~h}$ prior to the addition of WST-1 solution (EZ-Cytox cell viability assay kit; Itsbio, Seoul, Korea) for $1 \mathrm{~h}$. The optical density of the colored WST-1 was measured at $450 \mathrm{~nm}$ and normalized using optical density at $620 \mathrm{~nm}$. The results are expressed as the mean percentage \pm standard error (SE) of experiments performed in triplicate. Statistical significance was determined using a twotailed t-test compared with untreated nHDFs or UVB-exposed nHDFs not treated with troxerutin.

Cell cycle and DNA repair analyses. Cell cycle distribution was determined by measuring the DNA content. nHDFs were seeded in 60-mm culture dishes (SPL Life Sciences) at a density of $7 \times 10^{5}$ cells/dish. The cells were incubated for $24 \mathrm{~h}$ under growth conditions and then pre-treated with $10 \mu \mathrm{M}$ troxerutin for $8 \mathrm{~h}$. At various time points, the cells were exposed to $100 \mathrm{~mJ} / \mathrm{cm}^{2} \mathrm{UVB}$ and incubated for a further $24 \mathrm{~h}$. Following incubation, the nHDFs were fixed with $70 \%$ ethanol at $4{ }^{\circ} \mathrm{C}$ for $3 \mathrm{~h}$ and incubated with staining solution containing $50 \mu \mathrm{g} / \mathrm{ml}$ PI (Sigma-Aldrich), 0.5\% Triton X-100 (Bioshop, Burlington, Canada) and $100 \mu \mathrm{g} / \mathrm{ml}$ RNase (Bioshop) at $37^{\circ} \mathrm{C}$ for $1 \mathrm{~h}$. DNA content was measured using the FL2-H channel of a FACSCalibur flow cytometer (BD Biosciences, San Jose, CA, USA). DNA repair activity was determined by transfection with a damaged plasmid as previously described (21). Before transfection, the pGL3 luciferase reporter plasmids were damaged with $2,000 \mathrm{~J} / \mathrm{m}^{2} \mathrm{UVC}$ radiation. The $\mathrm{nHDF}$ were seeded at $7 \times 10^{5}$ cells in $60-\mathrm{mm}$ culture dishes and transiently transfected with either the control pGL3 or the damaged pGL3 plasmids along with pSV- $\beta$-galactosidase plasmid (as a transfection control). The transfected cells were treated with $10 \mu \mathrm{M}$ troxerutin for $24 \mathrm{~h}$. After the treatments, DNA repair activity was determined by using the Luciferase assay system (Promega, Madison, WI, USA). The results were normalized to $\beta$-galactosidase activity and are presented as the means \pm SE of the percentage of the control. Results shown are the averages of 3 independent experiments.

Migration assay. The migration rate was measured as described in a previous study (20). Briefly, $7 \times 10^{5} \mathrm{nHDFs}$ were seeded in a $60-\mathrm{mm}$ culture dish and grown to $>90 \%$ confluence. The cells were pre-treated with $10 \mu \mathrm{M}$ troxerutin for $8 \mathrm{~h}$ and then exposed to $100 \mathrm{~mJ} / \mathrm{cm}^{2} \mathrm{UVB}$. The cell monolayer was scraped in a straight line with a p200 pipet tip. At 0 and $48 \mathrm{~h}$ after wounding, the distance between the cells was compared by imaging using a phase contrast microscope.

miRNA microarray. Total RNA was extracted from each sample using RiboEX (GeneAll, Seoul, Korea) and labeled using Agilent miRNA labeling kits (Agilent Technologies, Santa Clara, CA, USA) according to the manufacturer's instructions. The labeled RNA was purified by a Micro Bio-Spin P-6 column (Bio-Rad Laboratories, Hercules, CA, USA) and hybridized with the SurePrint Human v16 miRNA microarray kit (based on miRBase Release 19.0; Agilent Technologies) as recommended by the manufacturer. The microarray contained 1,368 probes and detected 1,205 miRNAs. The fluorescence intensity of each probe was measured using Scanner and Feature Extraction software (Agilent Technologies). The digitalized fluorescence intensity was analyzed using GeneSpring GX version 11.5 (Agilent Technologies).

Bioinformatics analysis of miRNAs with significant changes in expression. Putative targets of miRNAs were identified using bioinformatics miRNA target prediction software, TargetScan (www.targetscan.org). The prediction of targets was performed using a conserved database for all miRNAs that were either upor downregulated by $>2$-fold. The targets of each significant miRNA were collected in a target pool and Gene Ontology (GO) of the target pool and were analyzed using DAVID (http:// david.abcc.ncifcrf.gov/). After GO analysis, the significant GO terms were filtered by a $>5 \%$ 'percentage of count', which represented the percentage of each GO term-related genes.

Statistical analyses. Statistical significance was determined by a Student's t-test. Values of $\mathrm{p}<0.05$ were considered to indicate statistically significant differences. 

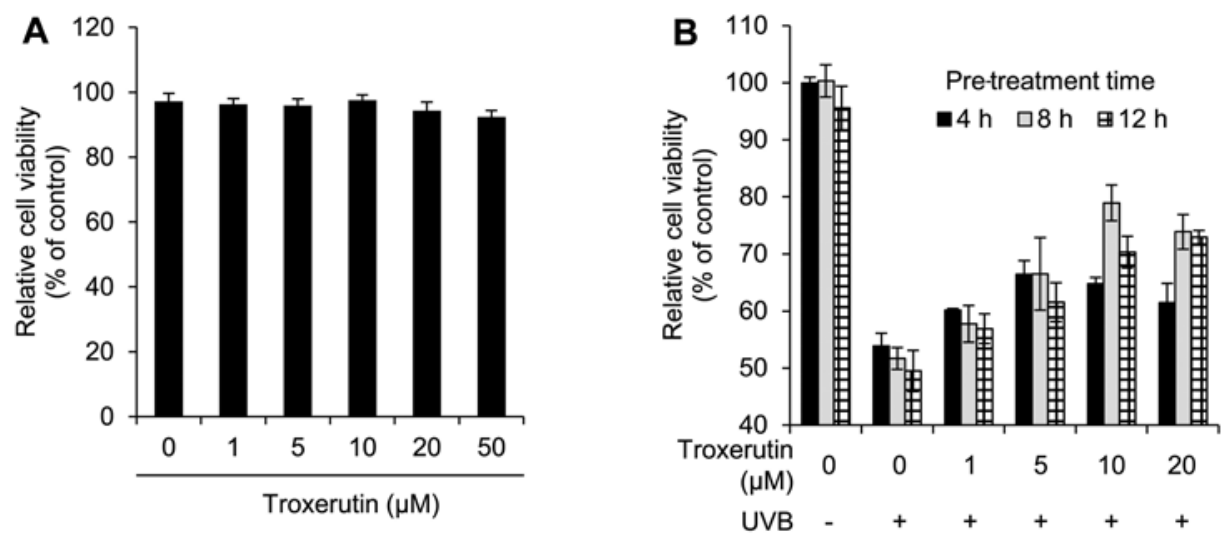

Figure 1. Troxerutin protects normal human dermal fibroblasts (nHDFs) against UVB-induced cytotoxicity. (A) Effects of troxerutin on nHDF viability. nHDFs were seeded in 96 -well plates and then treated with various concentrations of troxerutin $(0-50 \mu \mathrm{M})$ for $24 \mathrm{~h}$. (B) Protective effects of troxerutin against cytotoxicity induced by UVB radiation in nHDFs. nHDFs $\left(4 \times 10^{4}\right.$ cells/well) were seeded in 96 -well plates and pre-treated with $10 \mu \mathrm{M}$ troxerutin for 4,8 and $12 \mathrm{~h}$. Following pre-treatment, the nHDFs were exposed to $100 \mathrm{~mJ} / \mathrm{cm}^{2} \mathrm{UVB}$ and incubated for $24 \mathrm{~h}$. Cell viability was measured by WST-1 assay. The results are presented as the means \pm SE of the percentage of control optical density (OD) from experiments performed in triplicate.

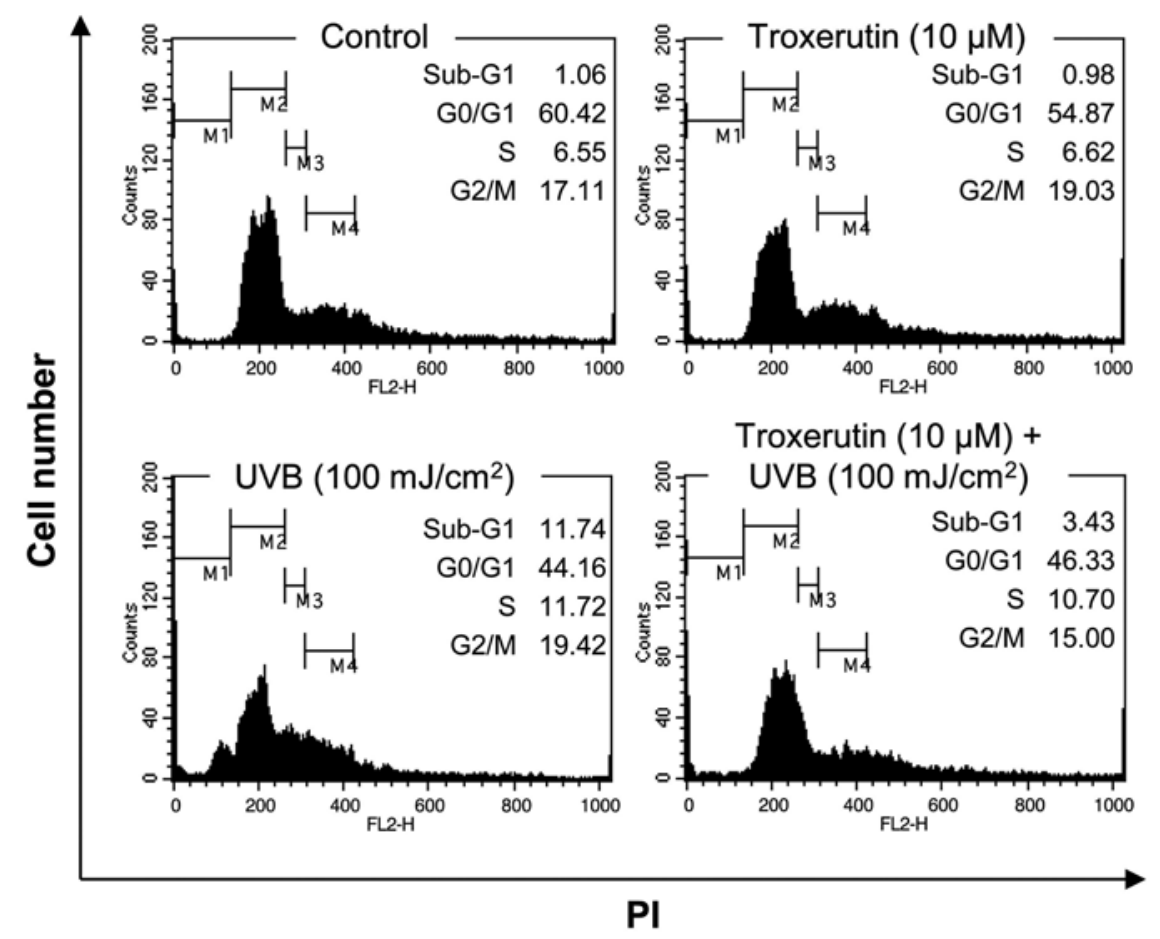

Figure 2. UVB-induced cell death was reduced by troxerutin. Normal human dermal fibroblasts (nHDFs; 7x10 $0^{5}$ cells) were seeded in 60 -mm culture dishes, treated with troxerutin for $8 \mathrm{~h}$, and then exposed to $100 \mathrm{~mJ} / \mathrm{cm}^{2} \mathrm{UVB}$. Follwoing treatment, the cells were incubated for $24 \mathrm{~h}$ and collected for PI staining of DNA. Fluorescence intensity distribution of the PI-stained cells was analyzed by a flow cytometer. Sub-G1, G1, S and G2/M phases were separated using the 4 indicated gates (M1, M2, M3 and M4).

\section{Results}

Cytotoxicity of troxerutin in $n H D F$ s and protective effects against $U V B$ radiation. To determine the cytotoxicity of troxerutin we first performed cell viability assays for nHDFs treated with the indicated concentrations of troxerutin. Overall, there was no significant toxicity observed for concentrations up to $50 \mu \mathrm{M}$ and treatment with $50 \mu \mathrm{M}$ troxerutin decreased cell viability to $92.40 \%$ (Fig. 1A). Therefore, we used troxerutin at concentrations $<50 \mu \mathrm{M}$ in the subsequent experiments.

We then investigated the protective effects of troxerutin against UVB radiation. nHDFs were pre-treated with 0-20 $\mu \mathrm{M}$ troxerutin for 4,8 and $12 \mathrm{~h}$, and then exposed to $100 \mathrm{~mJ} / \mathrm{cm}^{2}$ UVB. As shown in Fig. 1B, pre-treatment with troxerutin reversed the UVB-induced inhibitory effects on cell growth. In particular, pre-treatment with $10 \mu \mathrm{M}$ troxerutin for $8 \mathrm{~h}$ restored cell viability to $78.95 \%$ compared with $51.68 \%$ for the UVB-exposed nHDFs. We then examined whether troxerutin increases cell viability in nHDFs through the regulation of the cell cycle or cell death. To investigate cell cycle arrest we performed a flow cytometric cell cycle analysis using PI staining. As shown in Fig. 2, exposure to $100 \mathrm{~mJ} / \mathrm{cm}^{2} \mathrm{UVB}$ increased the number of cells in the sub-G1 phase, increasing the population of dead cells from 1.06 to $11.74 \%$. However, 

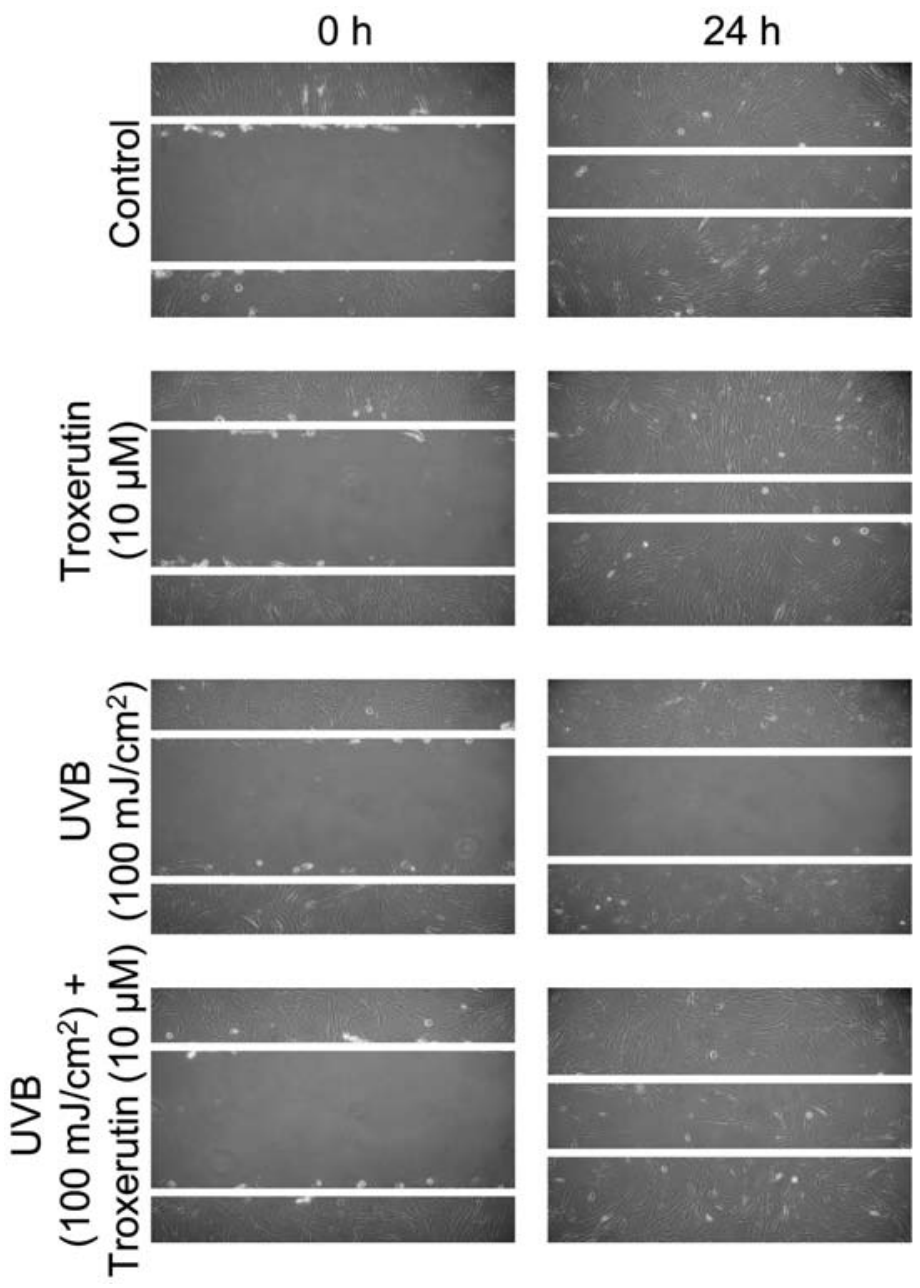

Figure 3. UVB-induced decrease in migration rate was reversed by troxerutin. Normal human dermal fibroblasts nHDFs (7x10 $0^{5}$ cells) were seeded in 60 -mm culture dishes, treated with troxerutin for $8 \mathrm{~h}$, and then exposed to $100 \mathrm{~mJ} / \mathrm{cm}^{2} \mathrm{UVB}$. Following exposure to UVB radiation, a wound was created by scratching the cell monolayer. At 0 and $24 \mathrm{~h}$, the wound was photographed under a phase contrast microscope (magnification, x200).

pre-treatment with $10 \mu \mathrm{M}$ troxerutin for $8 \mathrm{~h}$ reversed the UVB-induced increase in the number of cells in the sub-G1 phase (percentage of cells in sub-G1 phase decreased to 3.43\%). Therefore, troxerutin blocked the UVB-induced decrease in cell viability by repressing cell death.

Troxerutin enhances cell migration and DNA repair activity. To investigate the effects of troxerutin on the migration rate we performed a migration assay using a scratch line. nHDFs were grown to $>90 \%$ confluence and pre-treated with $10 \mu \mathrm{M}$ troxerutin for $8 \mathrm{~h}$. Following pre-treatment, the nHDFs were exposed to $100 \mathrm{~mJ} / \mathrm{cm}^{2} \mathrm{UVB}$ and scraped to form a scratch. As shown in Fig. 3, treatment with troxerutin enhanced the cell migration rate compared with the untreated (control) nHDFs and those exposed to UVB radiation and not treated with troxerutin. In addition, we examined whether troxerutin increases DNA repair activity. A plasmid containing the luciferase gene was damaged by UVC and then transfected into the nHDFs. The damaged vector was broken by endonucleases in the nHDFs, whereas the repaired vector remained and expressed luciferase, as shown in a previous study (21). Therefore, an increase in luciferase activity indicates enhanced DNA repair activity. As shown in Fig. 4, troxerutin increased luciferase activity in the nHDFs transfected with the damaged vector.

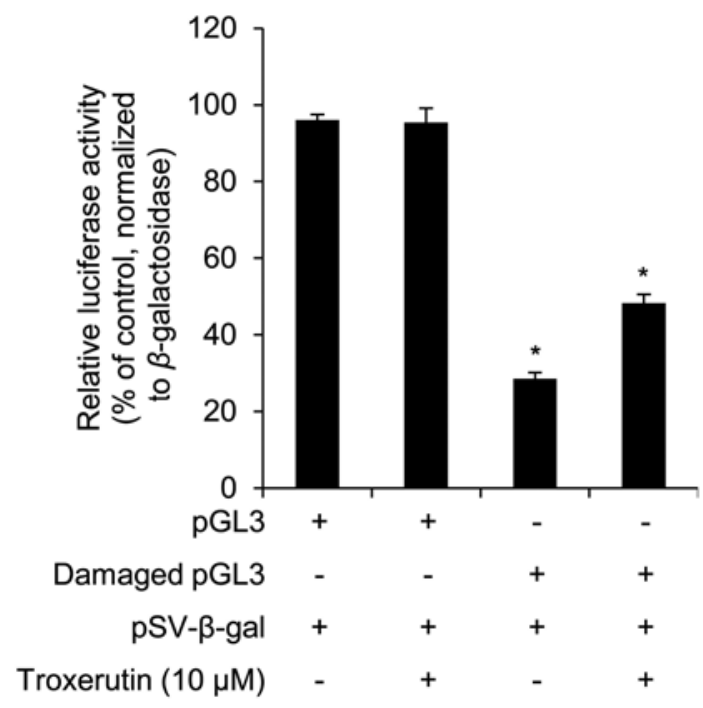

Figure 4. Troxerutin enhances the repair of DNA damage. Normal human dermal fibroblasts (nHDFs; $7 \times 10^{5}$ cells) were seeded in $60-\mathrm{mm}$ culture dishes and transfected with a damaged luciferase vector and pSV- $\beta$-gal. Following transfection, the cells were treated with $10 \mu \mathrm{M}$ troxerutin for $24 \mathrm{~h}$ and DNA repair activity was determined by luciferase activity. A $\beta$-galactosidase assay was performed for normalization. The results are presented as the means $\pm \mathrm{SE}$ of the percentage of luciferase activity in triplicate samples. Asterisks denote a significant difference compared with nHDFs transfected with the damaged pGL3 vector alone ( $\mathrm{p}<0.05)$. 


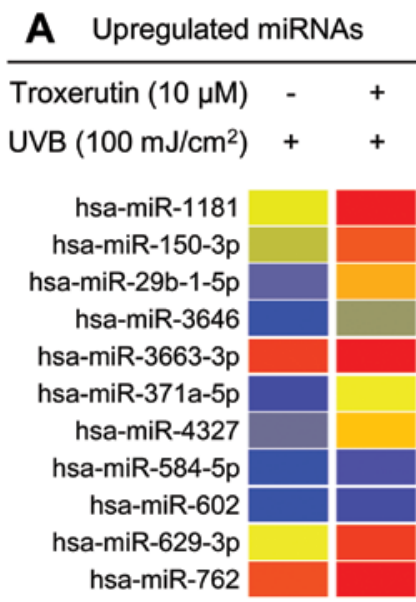

Expression level

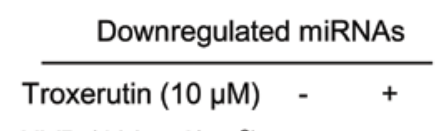

UVB $\left(100 \mathrm{~mJ} / \mathrm{cm}^{2}\right)++$
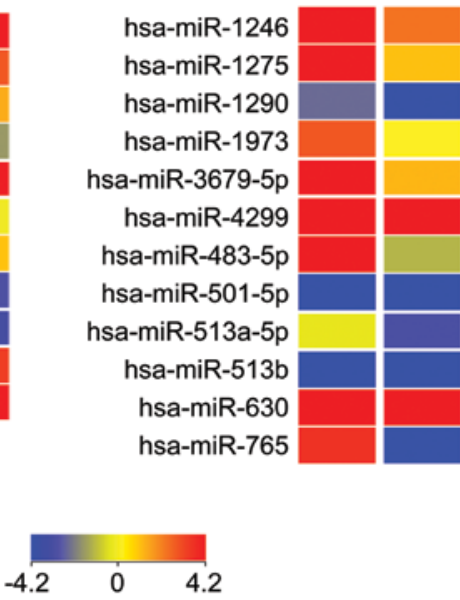

B

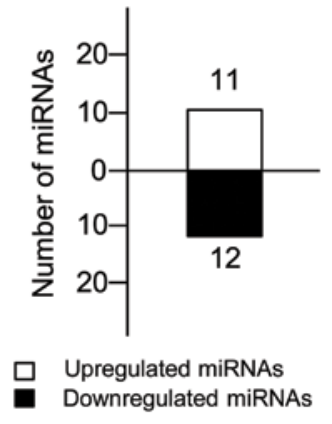

C

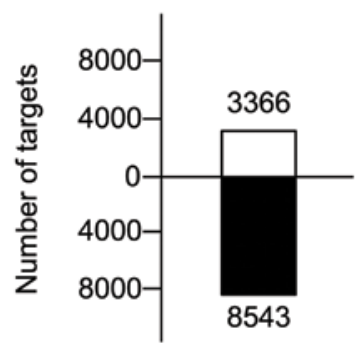

Targets of upregulated miRNAs

Target of downregulated miRNAs

Figure 5. Troxerutin alters miRNA expression profiles. (A) Heat map of troxerutin-regulated miRNAs. Normal human dermal fibroblasts (nHDFs; 7x10 5 cells) were seeded in 60-mm culture dishes, treated with troxerutin for $8 \mathrm{~h}$, and then exposed to $100 \mathrm{~mJ} / \mathrm{cm}^{2} \mathrm{UVB}$. Follwoing treatment with troxerutin and exposure to UVB radiation, miRNA expression was determined using miRNA microarray. Expression levels are indicated in the legend bar. (B) Total number of miRNAs that were either up- or downregulated by troxerutin in nHDFs. (C) Total number of targets of miRNAs that were either up- or downregulated by troxerutin in nHDFs.

Table I. Troxerutin-induced upregulated miRNAs in UVBexposed nHDFs.

\begin{tabular}{|c|c|c|c|}
\hline Accession no. & miRNA & $\begin{array}{c}\text { Fold } \\
\text { change }\end{array}$ & Chromosome \\
\hline MIMAT0005826 & hsa-miR-1181 & 3.45 & chr19 \\
\hline MIMAT0004610 & hsa-miR-150-3p & 2.82 & chr19 \\
\hline MIMAT0004514 & hsa-miR-29b-1-5p & 2.95 & $\operatorname{chr} 7$ \\
\hline MIMAT0018065 & hsa-miR-3646 & 2.29 & $\operatorname{chr} 20$ \\
\hline MIMAT0018085 & hsa-miR-3663-3p & 2.30 & $\operatorname{chr} 10$ \\
\hline MIMAT0004687 & hsa-miR-371a-5p & 2.43 & chr19 \\
\hline MIMAT0016889 & hsa-miR-4327 & 2.54 & $\operatorname{chr} 21$ \\
\hline MIMAT0003249 & hsa-miR-584-5p & 2.77 & $\operatorname{chr} 5$ \\
\hline MIMAT0003270 & hsa-miR-602 & 5.24 & $\operatorname{chr} 9$ \\
\hline MIMAT0003298 & hsa-miR-629-3p & 2.51 & $\operatorname{chr} 15$ \\
\hline MIMAT0010313 & hsa-miR-762 & 3.60 & chr16 \\
\hline
\end{tabular}

Identification of troxerutin-induced alterations in miRNA expression in $U V B$-exposed $n H D F s$ and target prediction of these miRNAs. We identified 23 troxerutin-specific miRNAs whose expression was altered by $>2$-fold in the troxerutintreated nHDFs (Fig. 5A and B), including 11 upregulated miRNAs (Table I) and 12 downregulated miRNAs (Table II). To elucidate the biological functions of the troxerutin-specific miRNAs, we identified putative targets using TargetScan, a seed sequence-based miRNA target prediction system. Since the miRNAs and miRNA target sites are conserved in various
Table II. Troxerutin-induced downregulated miRNAs in UVBexposed nHDFs.

\begin{tabular}{|c|c|c|c|}
\hline Accession no. & miRNA & $\begin{array}{c}\text { Fold } \\
\text { change }\end{array}$ & Chromosome \\
\hline MIMAT0005898 & hsa-miR-1246 & -3.31 & $\operatorname{chr} 2$ \\
\hline MIMAT0005929 & hsa-miR-1275 & -2.95 & chr6 \\
\hline MIMAT0005880 & hsa-miR-1290 & -2.32 & chr1 \\
\hline MIMAT0009448 & hsa-miR-1973 & -2.02 & chr4 \\
\hline MIMAT0018104 & hsa-miR-3679-5p & -3.20 & $\operatorname{chr} 2$ \\
\hline MIMAT0016851 & hsa-miR-4299 & -2.42 & chr11 \\
\hline MIMAT0004761 & hsa-miR-483-5p & -10.23 & $\operatorname{chr} 11$ \\
\hline MIMAT0002872 & hsa-miR-501-5p & -2.38 & $\operatorname{chrX}$ \\
\hline MIMAT0002877 & hsa-miR-513a-5p & -2.13 & $\operatorname{chrX}$ \\
\hline MIMAT0005788 & hsa-miR-513b & -2.49 & $\operatorname{chr} \mathrm{X}$ \\
\hline MIMAT0003299 & hsa-miR-630 & -3.86 & $\operatorname{chr} 15$ \\
\hline MIMAT0003945 & hsa-miR-765 & -13.79 & chr1 \\
\hline
\end{tabular}

species (22), we performed TargetScan in the conserved database. Through TargetScan, we identified 3,366 targets of troxerutin-specific upregulated miRNAs and 8,543 targets of troxerutin-specific downregulated miRNAs (Fig. 5C).

Biological functions of putative targets of troxerutin-specific miRNAs. To identify the biological functions of the putative targets of miRNAs that were either up- or downregulated in response to troxerutin, we performed GO analysis using DAVID and obtained the GO distribution of the putative targets. 
Table III. Gene Ontology (GO) analysis of potential target genes of troxerutin-regulated miRNAs.

GO no.

GO term

Percentage of count

Targets of upregulated miRNAs

GO:0045449

GO:0010604

GO:0043067

GO:0007049

Targets of downregulated miRNAs

GO:0045449

GO:0007242

GO:0006793

GO:0008104

GO:0010604

GO:0042127
Regulation of transcription

17.5

Positive regulation of macromolecule metabolic processes

Regulation of programmed cell death

Cell cycle

5.5

5.2

5.0

Regulation of transcription

15.9

Intracellular signaling cascade

7.6

Phosphorus metabolic processes

5.9

Protein localization

5.5

Positive regulation of macromolecule metabolic processes

5.4

Regulation of cell proliferation
As shown in Table III, GO analysis of the putative targets of troxerutin-specific upregulated miRNAs revealed the following distribution of biological functions: regulation of transcription (17.5\%, percentage of GO term-related gene in the putative target pool), positive regulation of macromolecule metabolic processes $(5.5 \%)$, the regulation of programmed cell death $(5.2 \%)$ and cell cycle $(5.0 \%)$. GO analysis of putative targets of troxerutin-specific downregulated miRNAs revealed the following distribution of biological functions: regulation of transcription $(15.9 \%)$, intracellular signaling cascades $(7.6 \%)$, phosphorus metabolic processes $(5.9 \%)$, protein localization $(5.5 \%)$, positive regulation of macromolecule metabolic processes (5.4\%) and the regulation of cell proliferation (5.0\%).

\section{Discussion}

In the present study, we investigated the protective effects of troxerutin against UVB radiation in nHDFs and showed that the beneficial effects of troxerutin were mediated by miRNAs. These findings provide new insight into the protective mechanisms against UVB-induced damage in dermal fibroblasts. UVB radiation is linked to the majority of skin disorders, including cancer, photoaging, sunburn and hyperpigmentation (23). In the dermis, UVB exposure induces disorders, such as inflammation and photoaging through cellular senescence and apoptosis (7,24-28). Thus, protection from UVB radiation is important to maintain the physiological functions of the dermis.

We first demonstrated that troxerutin markedly protected nHDFs from UVB-induced cell growth arrest and death, as indicated by the restoration of cell viability and an increase in the sub-G1 cell population following exposure to UVB radiation (Figs. 1B and 2). In addition, we demonstrated that troxerutin enhanced cell migration and increased DNA repair activity in the nHDFs (Fig. 4). Under the same conditions, our data indicated that troxerutin altered the expression of 23 miRNAs by at least 2-fold in UVB-exposed nHDFs.

The targets of several of these miRNAs have been reported in previous studies. The overexpression of miR-602 (5.24-fold upregulation by troxerutin) has been shown to induce prolifera- tion and to target RASSF1A and p73, a member of the p53 family that represses proliferation and induces cell cycle arrest (29). miR-483-5p (downregulated 10.23-fold by troxerutin) has been shown to suppress proliferation through interference with ERK1 translation (30). miR-630 (3.86-fold downregulation by troxerutin) has been shown to target BCL2, BCL2L2, YAP1 and IGF-1R $(31,32)$. BCL2 and BCL2L2, members of the pro-apoptotic BCL-2 family, repress intrinsic apoptosis by blocking the release of cytochrome $c$ from the mitochondria (33). YAP1 is a co-transcription factor that increases the expression of growth-stimulated protein (34). Lastly, IGF-1R has been implicated in growth factor-mediated cell growth in various cells (31). In addition, in cisplatin-induced apoptosis, the expression of miR-630 has been shown to be increased by p63, a member of the p53 tumor suppressor family (32). Thus, it is reasonable to suggest that troxerutin mediates its UVB protective effects through the regulation of the expression of these miRNAs.

We then demonstrated biological function by GO analysis of the putative targets of troxerutin-specific miRNAs using DAVID (Table III). The putative targets of the troxerutinspecific upregulated miRNAs were involved in 'regulation of transcription', 'positive regulation of macromolecule metabolic processes', 'regulation of programmed cell death' and 'cell cycle'. Targets of troxerutin-specific downregulated miRNAs were involved in 'regulation of transcription', 'intracellular signaling cascade', 'phosphorus metabolic processes', 'protein localization', 'positive regulation of macromolecule metabolic processes' and 'regulation of cell proliferation'. In particular, GO analysis revealed that the protective effects of troxerutin against UVB-induced cell death were mediated by alterations in the expression of miRNAs that targeted genes within specific functional categories, including programmed cell death, cell cycle and cell proliferation.

Overall, our study provides information on a number of putative miRNA-target associations. Further studies are required to mechanistically explore the involvement of these miRNAs in the protective effects of troxerutin against UVB-induced cell damage in nHDFs. In addition, the discovery of previously 
unidentified functional associations may lead to the development of novel therapeutic approaches in UVB-induced skin disorders.

\section{Acknowledgements}

We would like to thank all the other members of Coreana Cosmetics Co., Ltd. for their support. This study was supported by the KU Research Professor Program of Konkuk University and a grant from the Ministry of Science, ICT and Future Planning (grant no. 20110028646) of the Republic of Korea.

\section{References}

1. Brown RA, Prajapati R, McGrouther DA, Yannas IV and Eastwood M: Tensional homeostasis in dermal fibroblasts: mechanical responses to mechanical loading in three-dimensional substrates. J Cell Physiol 175: 323-332, 1998.

2. Sha W, Thompson K, South J, Baron M and Leask A: Loss of PPAR $\gamma$ expression by fibroblasts enhances dermal wound closure. Fibrogenesis Tissue Repair 5: 5, 2012.

3. Johansson S, Hedman K, Kjellén L, Christner J, Vaheri A and Höök M: Structure and interactions of proteoglycans in the extracellular matrix produced by cultured human fibroblasts. Biochem J 232: 161-168, 1985.

4. Quan T, He T, Kang S, Voorhees JJ and Fisher GJ: Solar ultraviolet irradiation reduces collagen in photoaged human skin by blocking transforming growth factor-beta type II receptor/Smad signaling. Am J Pathol 165: 741-751, 2004.

5. Uitto J: The role of elastin and collagen in cutaneous aging: intrinsic aging versus photoexposure. J Drugs Dermatol 7 (Suppl 2): S12-S16, 2008.

6. Yaar M and Gilchrest BA: Photoageing: mechanism, prevention and therapy. Br J Dermatol 157: 874-887, 2007.

7. Naik E, Michalak EM, Villunger A, Adams JM and Strasser A: Ultraviolet radiation triggers apoptosis of fibroblasts and skin keratinocytes mainly via the $\mathrm{BH} 3$-only protein Noxa. J Cell Biol 176: 415-424, 2007.

8. Vincent F, Deplanque G, Ceraline J, Duclos B and Bergerat JP: p53-independent regulation of cyclin B1 in normal human fibroblasts during UV-induced G2-arrest. Biol Cell 91: 665-674, 1999.

9. Cai Y, Yu X, Hu S and Yu J: A brief review on the mechanisms of miRNA regulation. Genomics Proteomics Bioinformatics 7 : 147-154, 2009.

10. Zhou BR, Xu Y, Permatasari F, et al: Characterization of the miRNA profile in UVB-irradiated normal human keratinocytes. Exp Dermatol 21: 317-319, 2012.

11. Zhou BR, Xu Y and Luo D: Effect of UVB irradiation on microRNA expression in mouse epidermis. Oncol Lett 3: 560-564, 2012.

12. Li W, Zhou BR, Hua LJ, Guo Z and Luo D: Differential miRNA profile on photoaged primary human fibroblasts irradiated with ultraviolet A. Tumour Biol: Jul 7, 2013 (Epub ahead of print).

13. Mancini M, Saintigny G, Mahé C, Annicchiarico-Petruzzelli M, Melino G and Candi E: MicroRNA-152 and -181a participate in human dermal fibroblasts senescence acting on cell adhesion and remodeling of the extra-cellular matrix. Aging 4: 843-853, 2012.

14. Sing T, Jinnin M, Yamane K, et al: microRNA-92a expression in the sera and dermal fibroblasts increases in patients with scleroderma. Rheumatology 51: 1550-1556, 2012.

15 . Honda N, Jinnin M, Kajihara I, et al: TGF- $\beta$-mediated downregulation of microRNA-196a contributes to the constitutive upregulated type I collagen expression in scleroderma dermal fibroblasts. J Immunol 188: 3323-3331, 2012.
16. An IS, An S, Park S, Lee SN and Bae S: Involvement of microRNAs in epigallocatechin gallate-mediated UVB protection in human dermal fibroblasts. Oncol Rep 29: 253-259, 2013.

17. Lu J, Wu DM, Zheng YL, Hu B, Cheng W, Zhang ZF and Li MQ Troxerutin counteracts domoic acid-induced memory deficits in mice by inhibiting CCAAT/enhancer binding protein $\beta$-mediated inflammatory response and oxidative stress. J Immunol 190: 3466-3479, 2013.

18. Ping X, Junqing J, Junfeng $\mathrm{J}$ and Enjin J: Radioprotective effects of troxerutin against gamma irradiation in mice liver. Int $\mathbf{J}$ Radiat Biol 88: 607-612, 2012.

19. Lu J, Wu DM, Zheng ZH, Zheng YL, Hu B and Zhang ZF: Troxerutin protects against high cholesterol-induced cognitive deficits in mice. Brain 134: 783-797, 2011.

20. Liang CC, Park AY and Guan JL: In vitro scratch assay: a convenient and inexpensive method for analysis of cell migration in vitro. Nat Protoc 2: 329-333, 2007.

21. Tran H, Brunet A, Grenier JM, et al: DNA repair pathway stimulated by the forkhead transcription factor FOXO3a through the Gadd45 protein. Science 296: 530-534, 2002.

22. Saetrom P, Snøve O, Nedland M, et al: Conserved microRNA characteristics in mammals. Oligonucleotides 16: 115-144, 2006.

23. Armstrong BK and Kricker A: The epidemiology of UV induced skin cancer. J Photochem Photobiol B 63: 8-18, 2001.

24. Son ED, Shim JH, Choi H, et al: Cathepsin G inhibitor prevents ultraviolet B-induced photoaging in hairless mice via inhibition of fibronectin fragmentation. Dermatology 224: 352-360, 2012.

25. Wang Q, Ye Y, Liu W, et al: Dual effects of silibinin treatment on autophagy-regulated dermal apoptosis retardation and epidermal apoptosis up-regulation in UVB-induced skin inflammation. J Asian Nat Prod Res 14: 688-699, 2012.

26. Hong MJ, Ko EB, Park SK and Chang MS: Inhibitory effect of Astragalus membranaceus root on matrix metalloproteinase-1 collagenase expression and procollagen destruction in ultraviolet $\mathrm{B}$-irradiated human dermal fibroblasts by suppressing nuclear factor kappa-B activity. J Pharm Pharmacol 65: 142-148, 2013.

27. Averbeck M, Gebhardt CA, Voigt S, et al: Differential regulation of hyaluronan metabolism in the epidermal and dermal compartments of human skin by UVB irradiation. J Invest Dermatol 127: 687-697, 2007.

28. Meeran SM, Akhtar S and Katiyar SK: Inhibition of UVB-induced skin tumor development by drinking green tea polyphenols is mediated through DNA repair and subsequent inhibition of inflammation. J Invest Dermatol 129: 1258-1270, 2009.

29. Yang L, Ma Z, Wang D, Zhao W, Chen L and Wang G: MicroRNA-602 regulating tumor suppressive gene RASSF1A is overexpressed in hepatitis B virus-infected liver and hepatocellular carcinoma. Cancer Biol Ther 9: 803-808, 2010.

30. Wang L, Shi M, Hou S, et al: MiR-483-5p suppresses the proliferation of glioma cells via directly targeting ERK1. FEBS Lett 586: 1312-1317, 2012.

31. Farhana L, Dawson MI, Murshed F, Das JK, Rishi AK and Fontana JA. Upregulation of miR-150* and miR-630 induces apoptosis in pancreatic cancer cells by targeting IGF-1R. PLoS One 8: e61015, 2013.

32. Huang Y, Chuang A, Hao H, et al: Phospho- $\Delta \mathrm{Np} 63 \alpha$ is a key regulator of the cisplatin-induced microRNAome in cancer cells. Cell Death Differ 18: 1220-1230, 2011.

33. Sasi N, Hwang M, Jaboin J, Csiki I and Lu B: Regulated cell death pathways: new twists in modulation of BCL2 family function. Mol Cancer Ther 8: 1421-1429, 2009.

34. Zhao B, Kim J, Ye X, Lai ZC and Guan KL: Both TEAD-binding and WW domains are required for the growth stimulation and oncogenic transformation activity of yes-associated protein. Cancer Res 69: 1089-1098, 2009. 